\title{
Study of magnetic field influence on magnetization distribution in three-layer ferromagnetic-antiferromagnetic spin systems
}

\author{
S. V. Belim \\ sbelim@mail.ru \\ Omsk State Technical University, 11 Mira Av., Omsk, 644050, Russia \\ Siberian State Automobile and Highway University, 4 Mira Av., Omsk, 644080, Russia
}

\begin{abstract}
In the paper three-layer spin systems from two ferromagnetic films separated by an antiferromagnetic film are examined. These systems are used in spintronics devices. Films described by the Ising model are considered. Ferromagnetic films are made of different materials. One ferromagnetic film is magnetic soft, the second is magnetically hard. The behavior of the system in the external magnetic field has been investigated. Computer simulation is performed using the Metropolis algorithm. Each film has its own order parameter. The finite dimension scaling theory is used to define model parameters. Systems with different ratio of exchange integrals are considered. The phase transition temperature was determined for each films using Binder cummulants. A phase diagram of the system is built at different ratios of exchange integrals. Phases have been identified that can be used in the design of spintronics devices. The phase transition temperature was found to be dependent on the film thickness. The magnetic field for magnetization switching of a soft magnetic film is determined as a function on temperature and the exchange integral. The maximum magnetic field for changing magnetization only one film is calculated. It is shown that as the temperature increases, the value of the minimum magnetic field for magnetization switching decreases. An increase in exchange integral value leads to an increase in the minimum value for magnetization switching magnetic field. For thin films, the dependence of the minimum magnetic field strength is weakly dependent on the film thickness. The values of the studied magnetic fields are considerably less than the limit magnetic field for the antiferromagnetic film and do not lead to suppression of the antiferromagnetic order.
\end{abstract}

Keywords: multilayer films, antiferromagnetic films, ferromagnetic films.

УДК: 537.622 .5

\section{Исследование влияния магнитного поля на распределение намагниченности в трехслойных ферромагнитно- антиферромагнитных спиновых системах}

\author{
Белим С. В. \\ Омский государственный технический университет, пр. Мира, 11, Омск, 644050, Россия \\ Сибирский государственный автомобильно-дорожный университет, пр. Мира 4, Омск, 644080, Россия
}

В статье проведено исследование трехслойных спиновых систем из двух ферромагнитных пленок, разделенных антиферромагнитной пленкой, используемых в устройствах спинтроники. Рассмотрены пленки, описываемые моделью Изинга. Ферромагнитные пленки выполнены из различных материалов. Одна ферромагнитная пленка является магнитомягкой, вторая - магнитотвердой. Исследовано поведение системы во внешнем магнитном поле. Компьютерное моделирование выполнено с помощью алгоритма Метрополиса. Для каждой из пленок введен свой параметр порядка. Для определения параметров модели использована теория конечноразмерного скейлинга. Рассмотрены системы с различным отношением обменных интегралов. Для каждой из пленок определена температура фазового перехода с помощью куммулянтов Биндера. Построена фазовая диаграмма системы при различных отношениях обменных интегралов. Показано, что введение антиферромагнитной пленки снижает связь между ферромагнитными пленками. Выделены фазы, которые могут быть использованы при проектировании устройств спинтроники. Найдена зависимость температуры фазового перехода от толщины пленки. Определена зависимость 
магнитного поля переключения намагниченности магнитомягкой пленки от температуры и значения обменного интеграла. Вычислено максимальное значение магнитного поля, приводящее к изменению намагниченности только одной пленки. Показано, что с ростом температуры уменьшается значение минимального магнитного поля переключения намагниченности. Увеличение значения обменного интеграла ведет к росту минимального значения напряженности магнитного поля переключения намагниченности. Для тонких пленок зависимость минимальной напряженности магнитного поля слабо зависит от толщины пленки. Значения исследуемых магнитных полей значительно меньше предельного магнитного поля для антиферромагнитной пленки и не приводит к подавлению антиферромагнитного порядка.

Ключевые слова: многослойные пленки, антиферромагнитные пленки, ферромагнитные пленки.

\section{1. Введение}

Интерес к исследованию многослойных спиновых систем обусловлен использованием в устройствах спинтроники, основанных на явлении гигантского магнитосопротивления $[1,2]$. Основной составляющей многослойных структур являются ферромагнитные пленки, разделенные слоем из немагнитного материала $[3,4]$. При этом присутствует взаимодействие между ферромагнитными слоями. Данное межслойное взаимодействие может носить как ферромагнитный, так и антиферромагнитный характер в зависимости от толщины прослойки. В любом случае, ферромагнитные слои взаимосвязанны. При реализации устройств спинтроники выбирают количество слоев так, чтобы межслойное взаимодействие носило антиферромагнитный характер. В этом случае, в отсутствии магнитного поля, ферромагнитные слои имеют противоположно направленные магнитные моменты. Такая ориентация намагниченности приводит к большому значению электрического сопротивления вследствие эффекта гигантского магнитосопротивления. Под действием внешнего магнитного поля одна из ферромагнитных пленок меняет знак намагниченности на противополжный, что приводит к резкому снижению электрического сопротивления.

Часто в такие системы добавляют антиферромагнитный слой поверх одной из ферромагнитных пленок для повышения устойчивости одного из слоев к внешнему магнитному полю. Такие системы получили название спиновых вентилей $[5,6]$. Добавление антиферромагнитного слоя эффективно при условии значительно большего значения обменного интеграла в антиферромагнетике по сравнению с ферромагнетиком. Компьютерное моделирование методом Монте-Карло многослойных систем было осуществлено в работах $[7,8]$.

В последнее время активно стало развиваться направление, называемое антиферромагнитной спинтроникой [9], в котором антиферромагнитные пленки используются для прослоек между ферромагнитными слоями и для реализации двухслойных систем ферромагнетик/антиферромагнтик $[10,11]$

Целью данной статьи является исследование распределения намагниченности в трехслойных структурах, описываемых моделью Изинга, состоящих из двух ферромагнитных пленок, разделенных антиферромагнитной пленкой, во внешнем магнитном поле.

\section{2. Описание системы}

Рассмотрим систему, состоящую из трех пленок. Две крайние пленки из ферромагнитных материалов толщиной $D$ разделены пленкой из антиферромагнитного материала толщиной $d$. В рамках компьютерного моделирования толщина интерпретируется как количество спиновых слоев. Будем считать, что пленки расположены параллельно плоскости OXY. Ограничимся случаем двух возможных состояний спинов системы $(S=1 / 2$ или $S=-1 / 2)$, который описывается моделью Изинга. Также будем рассматривать системы с различными значениями обменных интегралов в ферромагнитных слоях. В первом ферромагнитном слое $(0 \leq z<D)$ значение обменного интеграла $J$, в антиферромагнитном слое $(D \leq z<D+d)$ обменный интеграл $J_{a}$, во втором ферромагнитном слое $(D+d \leq z<2 D+d)$ обменный интеграл $J_{1}$. Причем выполняется неравенство $J \geq J_{1}$. Поэтому первый ферромагнитный слой в дальнейшем будем называть магнитожестким, а второй - магнитомягким.

Гамильтониан такой системы во внешнем магнитном поле запишется в виде:

$$
H=-J \sum_{0 \leq z<D} S_{i} S_{j}+J_{a} \sum_{D \leq z<D+d} S_{i} S_{j}-J_{1} \sum_{D+d \leq z<2 D+d} S_{i} S_{j}+\mu h_{0} \sum S_{i} .
$$

Здесь $S_{i}-$ спин в $i$-ом узле, $h_{0}-$ напряженность магнитного поля, $\mu-$ магнетон Бора. Перейдем к безразмерным относительным величинам:

$$
R_{a}=J_{a} / J, R_{1}=J_{1} / J, h=\mu h_{0} / J .
$$

В результате получаем следующее выражение для Гамильтониа:

$$
H / J=-\sum_{0 \leq z<D} S_{i} S_{j}+R_{a} \sum_{D \leq z<D+d} S_{i} S_{j}-R_{1} \sum_{D+d \leq z<2 D+d} S_{i} S_{j}+h \sum S_{i} .
$$

Для температуры системы $t$ также введем безразмерную величину $T=k t / J$, где $k-$ постоянная Больцмана.

Прежде всего необходимо построить фазовую диаграмму системы, для определения параметров, при которых слои системы находится в ферромагнитном состоянии. Для описания магнитных свойств системы введем четыре параметра порядка. Намагниченность магнитотвердой пленки обозначим через $m_{1}$, а магнитомягкой через $-m_{2}$. Обе эти величины вычисляются как простая сумма значений спинов в единице объема системы. Для исследования антиферромагнитного упорядочивания в средней пленке введем параметр по- 
рядка $m_{a}$, который вычисляется как разность магнитных моментов подрешеток с четными и нечетными суммами индексов в единице объема.

$$
m_{a}=\sum_{\text {even }} S_{i}-\sum_{\text {odd }} S_{i}
$$

Компьютерное моделирование осуществлялось для трехслойных систем с линейными размерами пленок $L \times L$. Система размещалась между двумя плоскостями $z=0$ и $z=2 D+d-1$. Вдоль направлений осей $O X$ и $O Y$ использовались периодические граничные условия. Компьютерное моделирование осуществлялось с помощью алгоритма Метрополиса [12]. Для моделирования был реализован программный комплекс с использованием технологии CUDA вычислений на графическом процессоре.

Для определения температур фазовых переходов использовались куммулянты Биндера четвертого порядка [15]:

$$
U_{1}=1-\frac{\left\langle m_{1}^{4}\right\rangle}{3\left\langle m_{1}{ }^{2}\right\rangle^{2}}, U_{2}=1-\frac{\left\langle m_{2}{ }^{4}\right\rangle}{3\left\langle m_{2}{ }^{2}\right\rangle^{2}}, U_{a}=1-\frac{\left\langle m_{a}{ }^{4}\right\rangle}{3\left\langle m_{a}{ }^{2}\right\rangle^{2}} .
$$

Угловыми скобками обозначено термодинамическое усреднение по состояниям системы. Согласно теории конечноразмерного скейлинга [13] значения куммулянтов Биндера при температуре фазового перехода не зависят от размеров системы. Для определения температуры фазового перехода необходимо построить графики зависимости куммулянтов Биндера для систем различного размера. Точка пересечения графиков соответствует температуре фазового перехода. Для построения фазовой диаграммы необходимо определить температуры фазовых переходов $T_{1}$ и $T_{2}$ для ферромагнитных пленок и температуру $T_{N}$ для антиферромагнитной пленки. Температуры ферромагнитных переходов вычислялись на основе куммулянтов $U_{1}$ и $U_{2}$, а температура антиферромагнитного перехода - на основе куммулянта $U_{a}$.

\section{3. Результаты компьютерного моделирования}

Компьютерный эксперимент проводился для систем с линейными размерами от $L=20$ до $L=36$ с шагом $\Delta L=4$. Изучались системы с толщиной ферромагнитных пленок $D=4, D=6$ и $D=8$. Толщина антиферромагнитной пленки также принимала значения $d=4, d=6$ и $d=8$. Рассматривались системы с различными комбинациями значений $D$ и $d$. Отношения обменных интегралов $R_{a}$ и $R_{1}$ принимали значения от 0.5 до 1.0 с шагом 0.1 . Для каждой комбинации толщин пленок строилась фазовая диаграмма системы при нулевом внешнем магнитном поле $h=0$. Расчеты показали, что общий вид фазовой диаграммы остается неизменным при различных значениях толщины пленки. Фазовая диаграмма для системы с $D=d=4$ и $R_{a}=0.8$ приведена на Рис. 1 .

Магнитотвердая ферромагнитная пленка может находиться в одной из фаз $F-$ ферромагнитная, $P-$ парамагнитная, магнитомягкая пленка - также в одной из фаз: $f-$ ферромагнитная, $p$ - парамагнитная. Аналогично можно выделить две фазы для антиферромагнитной пленки: $A-$ антиферромагнитная, $N-$ парамагнитная. В соответствии с этим на фазовой диаграмме присутствуют следующие фазы: $P / N / p, F / N / p, F / A / p$, $F / A / f$ и $F / N / f$. Для дальнейшего исследования интерес представляет фаза, в которой обе ферромагнитные пленки находятся в ферромагнитной фазе, то есть $F / A / f$ или $F / N / f$.

При изменении толщины пленки происходит повышение температур фазовых переходов. График зависимости температуры в магнитотвердой фазе от толщины пленки представлен на Рис. 2. Следует отметить, что температура антиферромагнитного перехода изменяется по такому же закону. Температура фазового перехода магнитомягкой пленки также увеличивается пропорционально во столько же раз, как и температура магнитотвердой пленки.

Для реализации спинового вентиля на основе гигантского магнитосопротивления необходимо, чтобы две пленки находились в ферромагнитной фазе с ориентацией намагниченности в противоположные стороны. Рассмотрим состояние системы, в котором две крайние пленки находятся в ферромагнитном состоянии. Приложим внешнее магнитное поле для перемагничивания магнитомягкого материала. При этом внешнее магнитное поле не должно изменять направление намагниченности магнитотвердой пленки. Также необходимо учитывать влияние внешнего магнитного поля на антиферромагнитную пленку. Как известно [14,15] внешнее магнитное поле подавляет антиферромагнитную фазу и в пленке может наблюдаться ферромагнитное упорядочение.

В рамках компьютерного моделирования определялись минимальное $H_{0}$ и максимальное $H_{1}$ значение напряженности магнитного поля, приводящее к изменению намагниченности магнитомягкой пленки без изменения знака намагниченности магнитотвердой пленки. Исследовалась зависимость величин $H_{0}$ и $H_{1}$ от температуры $T$, отношения обменных интегралов $R$ и толщин

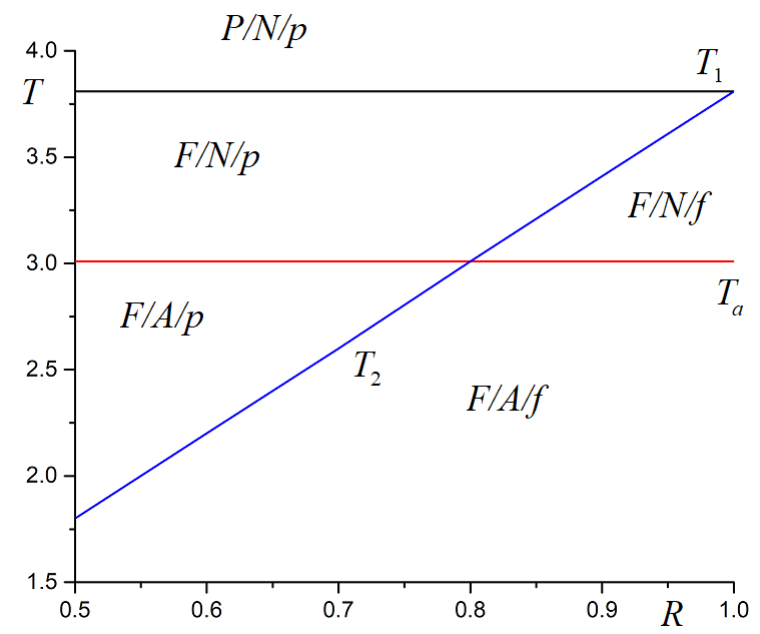

Рис. 1. Фазовая диаграмма системы для системы с $D=d=4$ и $R_{a}=0.8$.

Fig. 1. Phase diagram of system for case $D=d=4$ and $R_{a}=0.8$. 
пленок $D$. Зависимость величин магнитного поля $H_{0}$ и $H_{1}$ от температуры $T$ при различных значениях отношения обменных интегралов $R$ для пленок толщиной $D=4$ представлена на Рис. 2.

Как видно из Рис. 3 величина минимальной напряженности магнитного поля $H_{0}$ убывает с ростом температуры. Эта закономерность может быть объяснена тем, что величина намагниченности системы также убывает с ростом температуры при приближении к точке фазового перехода. В пределах исследованных значений толщин пленок значения напряженностей магнитных полей $H_{0}$ и $H_{1}$ не зависят от толщины пленки $D$ при температурах значительно ниже температуры фазового перехода и слабо зависят от температуры при приближении к точке фазового перехода.

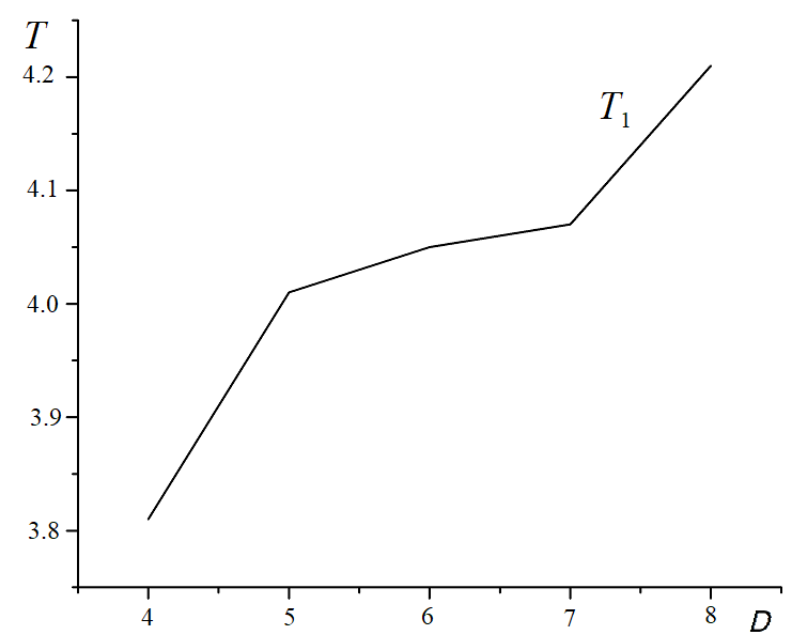

Рис. 2. Зависимость температуры фазового перехода магнитотвердой пленки $T_{1}$ от толщины $D$.

Fig. 2. The dependence of the phase transition temperature for the magneto-hard film $T_{1}$ on the thickness $D$.

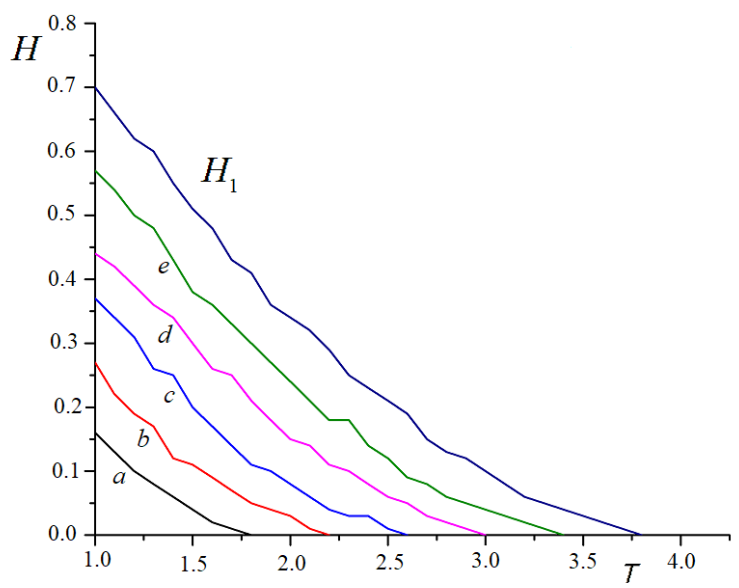

Рис. 3. Зависимость величин магнитного поля $H_{0}$ и $H_{1}$ от температуры $T$ для пленок толщиной $D=4$ при различных значениях отношения обменных интегралов: $R=0.5$ (a), $R=0.6(\mathrm{~b}), R=0.7$ (c), $R=0.8(\mathrm{~d}), R=0.9$ (e).

Fig. 3. Dependence the magnetic field $H_{0}$ and $H_{1}$ on temperature $T$ for films with thickness $D=4$ at different values of exchange integrals ratio: $R=0.5$ (a), $R=0.6$ (b), $R=0.7$ (c), $R=0.8$ (d), $R=0.9$ (e).

\section{4. Заключение}

Исходя из результатов компьютерного эксперимента можно сделать вывод о том, что введение антиферромагнитной пленки в качестве разделителя между двумя ферромагнитными пленками вместо немагнитного материала изменяет поведение системы. Этот результат согласуется с экспериментальными данными, описанными в статье [16] для многослойных систем $[\mathrm{Ni}-\mathrm{Fe}(60 \mathrm{нм}) / \operatorname{Ir}-\mathrm{Mn}(35$ нм)]×5. Прежде всего, наблюдается эффект снижения взаимосвязи между ферромагнитными слоями. Этот результат ранее был описан в статье [17] на основе экспериментальных данных для многослойных систем Nd-Dy-Fe-Co-B/a-Fe с разделительными слоями $\mathrm{Ta}, \mathrm{MgO}, \mathrm{Cr}_{2} \mathrm{O}_{3}$. Как следствие, в системах с антиферромагнитным разделительным слоем появляется дополнительная возможность управления направлением намагниченности ферромагнитных пленок, что важно для устройств спинтроники.

Также из результатов моделирования можно сделать вывод о нелинейной зависимости поля перемагничивания магнитомягкого слоя от температуры. Этот вывод согласуется с результатами экспериментальной работы [18] для трехслойных структур на основе Ni. Также нелинейная зависимость намагниченности от внешнего магнитного поля выявлена в статье [19] для многослойных структур с антиферромагнитным слоем $\left[\mathrm{Co}_{2} \mathrm{Fe}\left(\mathrm{Al}_{0.5} \mathrm{Si}_{0.5}\right)(\mathrm{CFAS}) / \mathrm{Ag}\right](\mathrm{N})$.

Благодарности/Aknowledgements. Исследование выполнено при финансовой поддержке РФФИ в рамках научного проекта № 20-07-00053. / The reported study was funded by RFBR, project number 20-07-00053.

\section{Литература/References}

1. M. N. Baibich, J. M. Broto, A. Fert, F. N. Van Dau, F. Petroff, P. Etienne, G. Creuzet, A. Friederich, J. Chazelas. Phys. Rev. Lett. 61, 2472 (1988). Crossref

2. A. Barthelemy, A. Fert. Phys. Rev. B. 43, 13124 (1991). Crossref

3. Y. Li, K. Baberschke. Phys. Rev. Lett. 68, 1208 (1992). Crossref

4. F. Huang, M. T. Kief, G. J. Mankey, R. F. Willis. Phys. Rev. B. 49, 3962 (1994). Crossref

5. C. H. Chen, Y. H. Cheng, C. W. Ko, W. J. Hsueh. Appl. Phys. Lett. 107, 152401 (2015). Crossref

6. A. Vedyayev, N. Ryzhanova, B. Dieny, N. Strelkov. Phys. Lett. A. 355, 243 (2006). Crossref

7. E. Birsan. Acta Physica Polonica A. 117, 500 (2010). Crossref

8. V. V. Prudnikov, P. V. Prudnikov, D. E. Romanovskii. Jetp Lett. 102, 668 (2015). Crossref

9. Ch. Song, Y. You, X. Chen, X. Zhou, Y. Wang, F. Pan. Nanotechnology. 29, 112001 (2018). Crossref

10. H. Sato, S. Saito, S. Mizuno, K. Akahane, H. Uchida. Electronics and Communications in Japan. 198, 27 (2017). Crossref

11. X. Qi, F. Hao, X. Li, Y. Li, Z. Lu. AIP Advances. 9, 105008 (2019). Crossref 
12. M. Weigel. International Journal of Modern Physics C. 23 (8), 1240002 (2012). Crossref

13. D. P. Landau, K. Binder. Phys. Rev. B. 17, 2328 (1978). Crossref

14. S. V. Belim, E. V. Trushnikova. Journal of Physics: Conf. Series. 1210, 012011 (2019). Crossref

15. S. V. Belim, E. V. Trushnikova. Letters on Materials. 8 (4), 440 (2018). Crossref

16. U. Kilic, C. A. Ross, C. Garcia. Phys. Rev. Appl. 10, 034043 (2018). $\underline{\text { Crossref }}$
17. Z. M. Dai, W. Liu, X. T. Zhao, Z. Han, D. Kim, C. J. Choi, Z. D. Zhang. J. Appl. Phys. 120 (16), 163906 (2016). Crossref

18. J. G. Choi, B. U. Kang, S. S. Lee, P. Khajidmaa. Journal of the Korean Magnetic Society. 29 (2), 42 (2019). Crossref

19. T. Shiroyama, Y. Sakuraba, T. Nakatani, H. SepehriAmin, J. M. Jung, K. Hono. J. Appl. Phys. 124 (16), 163910 (2018). $\underline{\text { Crossref }}$ 